\title{
Dimensional Variations of General Curved Composite Parts
}

\section{Chensong Dong}

\author{
Curtin University of Technology \\ GPO Box 1987, Perth, WA 6845, Australia \\ c.dong@curtin.edu.au
}

Keywords: composites; processing; dimensional variation.

\begin{abstract}
With the increasing demands of energy efficiency and environment protection, composite materials have become an important alternative for traditional materials. Composite materials offer many advantages over traditional materials including: low density, high strength, high stiffness to weight ratio, excellent durability, and design flexibility. Despite all these advantages, composite materials have not been as widely used as expected because of the complexity and cost of the manufacturing process. One of the main causes is associated with poor dimensional control. General curved composite parts are often used as the structural components in the composite industry. Due to the anisotropic material nature, process-induced dimensional variations make it difficult for tighttolerance control and limit the use of composites.

This research aims to develop a practical approach for the design of general curved composite parts and assembly. First, the closed-form solution for the process-induced dimensional variations, which is commonly called spring-in, was derived. For a general curved composite part, a Structural Tree Method (STM) was developed to divide the curve into a number of pieces and calculate the dimensional variations sequentially. This method can be also applied to an assembly of composite parts. The approach was validated through a case study. The method presented in this paper provides a convenient and practical tool for the dimensional and tolerance analysis in the early design stage of general curved composite parts and assembly, which is extremely useful for the realization of affordable tight tolerance composites. It also provides the foundation of Integrated Product/Process Development (IPPD) and Design for Manufacturing/Assembly (DFM/DFA) for composites.
\end{abstract}

\section{Introduction}

The last four decades have seen a tremendous advancement in the science and technology of fiber reinforced composites. The low density, high strength, high stiffness to weight ratio, excellent durability, and design flexibility of composites are the primary reasons for their use in many structural components in aircraft, automotive, marine, and other industries [1]. With the increasing demands of energy efficiency and environment protection, composite materials will continue to find wide applications. General curved parts are one of the most commonly used structural components in the composite industry.

Despite all these advantages, composite materials have not been as widely used as expected because of the complexity and cost of manufacturing process. One of the main causes is associated with poor dimensional control. For example, NASA is investigating the feasibility of SCRIMP to produce aircraft quality heavily-loaded primary structures. Additional technology development is required to achieve dimensional control and acceptable fiber volume fraction for thick structural elements [2].

Unlike processing of metal materials, in composite processing, material and part form at the same time. Typical manufacturing processes are injecting resin into the preform of fiber in the mold and curing at some temperature (often higher than room temperature), such as RTM and VARTM 
processes. When the composite part is being cured, resin shrinks due to the chemical reaction (crosslinking). After curing, the part is cooled down to the room temperature. Due to the anisotropic material nature, dimensional variations are induced by the fiber-resin CTE mismatch and the curing shrinkage of resin.

For symmetric laminates, spring-in is a common problem in composite processing, as shown in Fig. 1. Hahn and Pagano [3] performed an elastic analysis of the residual stresses in a thermoset matrix composite. Radford and Diefendorf [4] developed a simple mathematical formula to predict the spring-in of curved shaped parts. Their formula was used by Huang and Yang [5] in their experimental studies. Kollar [6] presented an approximate analysis of spring-in. Jain and Mai [7, 8] developed a mechanics-based model using modified shell theory. Yoon and Kim [9] developed a computational method by applying the characterized properties to the classical lamination theory. Simple geometric structures such as "L-shape" were studied. In order to predict the spring-in of more complex structures, numerical simulation tools of finite element method or finite difference method are often employed. Wang et al. [10] conducted a finite element analysis of spring-in using ABAQUS. Ding et al. [11] developed a 3-D finite element analysis procedure to predict spring-in resulting from anisotropy for both thin and thick angled composite shell structures. It was concluded that the FE model gives more accurate results than the analytical model by Jain et al. [7], especially for thicker composite shells. It shows from the literature that although some studies have been carried out on the residual stress development in the composite processing, the dimensional variations have not been thoroughly studied. Most composite structures are simple geometry components such as "L-shape". The dimensional variations of complex geometries are not addressed. In addition, systematic studies on the tolerance analysis and synthesis for assemblies of composites are lacking. Thus, it is highly desirable to develop an approach to predict dimensional variations during the early design stage of composite components and assembly.

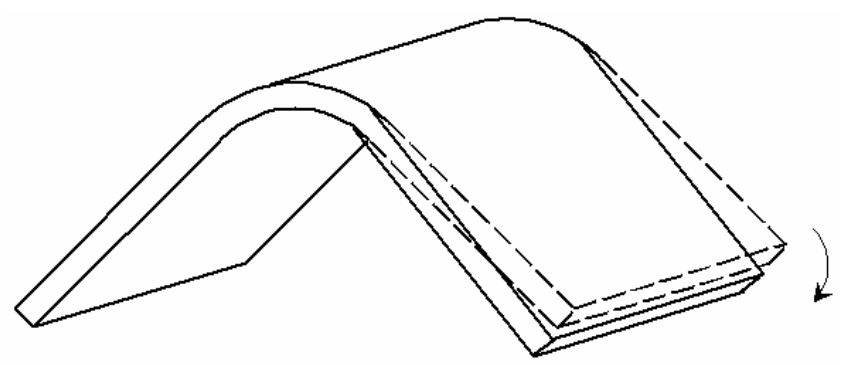

Fig. 1: Spring-in of an L-shape structure

This research aims to develop a practical approach for the design of general curved composite parts and assembly. The spring-in was calculated based on a simple mathematical formula and equivalent material properties. For a general curved composite part, a Structural Tree Method (STM) was developed to divide the curve into a number of pieces and calculate the dimensional variations sequentially. This method can be also applied to an assembly of composite parts. The approach was validated through a case study.

\section{Spring-in Model}

As shown in Fig. 2, the mechanism of spring-in is illustrated by using an L-shape structure. The included angle is denoted as $\phi$ and the radius of the curved section is denoted as $r$. The original arc length is given by

$$
s=r(180-\phi) \text {. }
$$

When the part is cooled down from the curing temperature to the room temperature, the temperature change is denoted as $\Delta T$ (negative). The radius and arc length become

$$
r^{\prime}=r\left(1+\alpha_{T} \Delta T\right) \text {. }
$$




$$
s^{\prime}=r(180-\phi)\left(1+\alpha_{I} \Delta T\right)
$$

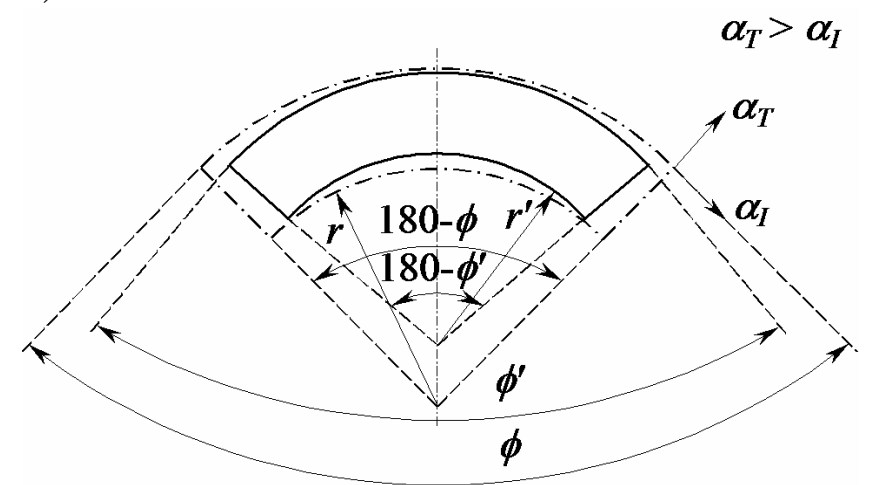

Fig. 2: Spring-in of an L-shape structure

where $\alpha_{I}$ and $\alpha_{T}$ are the in-plane and through-thickness CTEs, respectively. The included angle is given by

$$
\phi^{\prime}=180-\frac{s^{\prime}}{r^{\prime}}=180-(180-\phi) \frac{1+\alpha_{I} \Delta T}{1+\alpha_{T} \Delta T} .
$$

The spring-in is given by

$$
\Delta \phi=\phi^{\prime}-\phi=(180-\phi) \frac{\left(\alpha_{T}-\alpha_{I}\right) \Delta T}{1+\alpha_{T} \Delta T} \text {. }
$$

Eq. 5 shows that because of the mismatch of the in-plane CTE $\alpha_{I}$ and the through-thickness CTE $\alpha_{T}$, the included angle decreases after the composite part is cured, which is commonly called springin. The CTE mismatch is dependent on the material properties of fiber, resin, the curing shrinkage of resin, and the stacking sequence.

After the in-plane and through-thickness CTEs are known, the spring-in angle can be calculated using Eq. 5. Since $1+\alpha_{T} \Delta T \approx 1$, Eq. 5 can be simplified as

$$
\Delta \phi=(180-\phi)\left(\alpha_{T}-\alpha_{I}\right) \Delta T \text {. }
$$

Eq. 6 was validated against the data from literature to prove its accuracy. First, it was validated against the data from [6]. For an L-shaped [0] $]_{4}$ glass/epoxy laminate, i.e. $\phi=90^{\circ}$, when the temperature drop is $130^{\circ} \mathrm{C}$, the spring-in calculated from Eq. 6 is $-0.16^{\circ}$, which is in a good agreement with the theoretical result presented from [6]. Second, it was validated against the theoretical and experimental results from [7] and [8]. The spring-in angles of the carbon/epoxy laminates calculated by Eq. 6 are $-1.71^{\circ}$ for 0 laminates and $-1.31^{\circ}$ for $90^{\circ}$ laminates, respectively, which are in a good agreement with $-1.72^{\circ}$ and $-1.30^{\circ}$ from [7] and [8]. Thus, for the purpose of general composites design, Eq. 6 provides sufficient computational accuracy.

\section{Dimensional Variations of General Curved Parts}

For general curved parts, the dimensional variations due to spring-in could not be calculated from Eq. 6 straitforwardly. In order to calculate dimensional variations, a piecewise method is needed. This approach is illustrated through a general curved part as shown in Fig. 3.

For convenience, the concept of bending angle is introduced. For each arc piece, the bending angle is defined as $\beta=180-\phi$. Thus, Eq. 6 is written as

$$
\Delta \phi=\beta\left(\alpha_{T}-\alpha_{I}\right) \Delta T \text {. }
$$

The bending angle can be positive or negative. As shown in Fig. 4(a), the spring-in causes a counter-clockwise rotation, and the bending angle is defined as positive. Likewise, as shown in Fig. 4(b), the spring-in causes a clockwise rotation, and the bending angle is defined as negative. This is in accordance to the Cartesian tradition. If the starting and end points of a general curve is denoted as $P_{i-1}$ and $P_{i}$, respectively, the bending angle is given by 
$\beta=\arctan \tau_{i-1}-\arctan \tau_{i}$

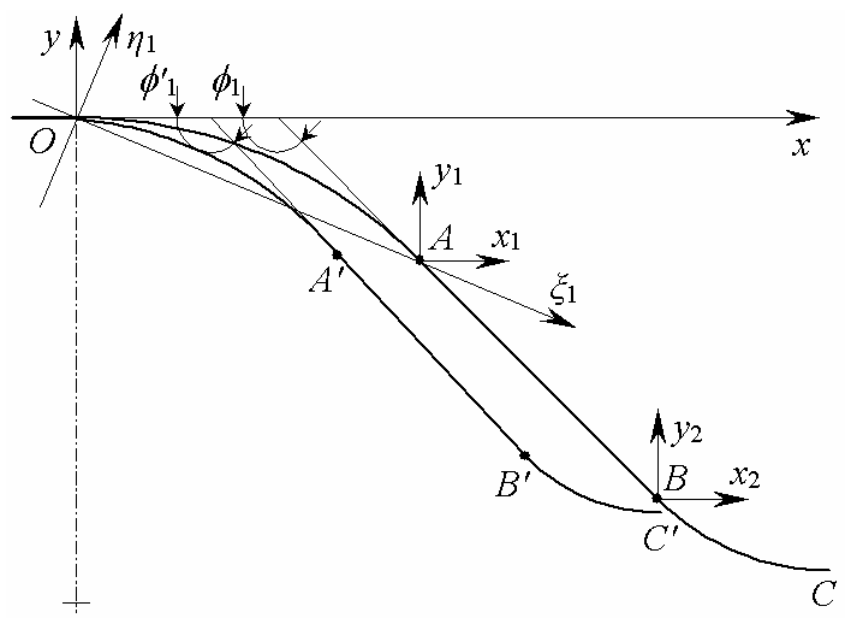

Fig. 3: Dimensional variations of a general curved part

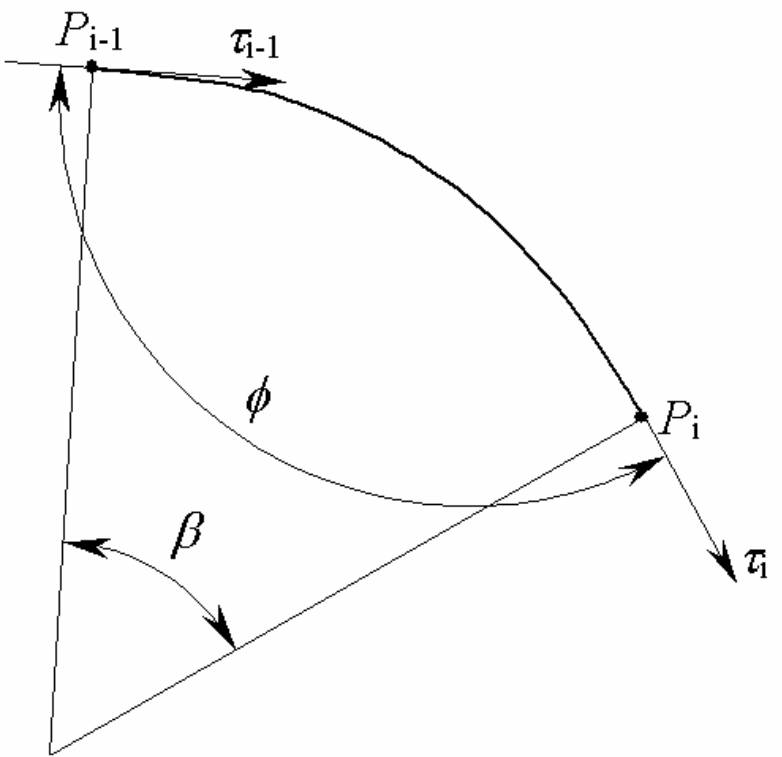

(a)

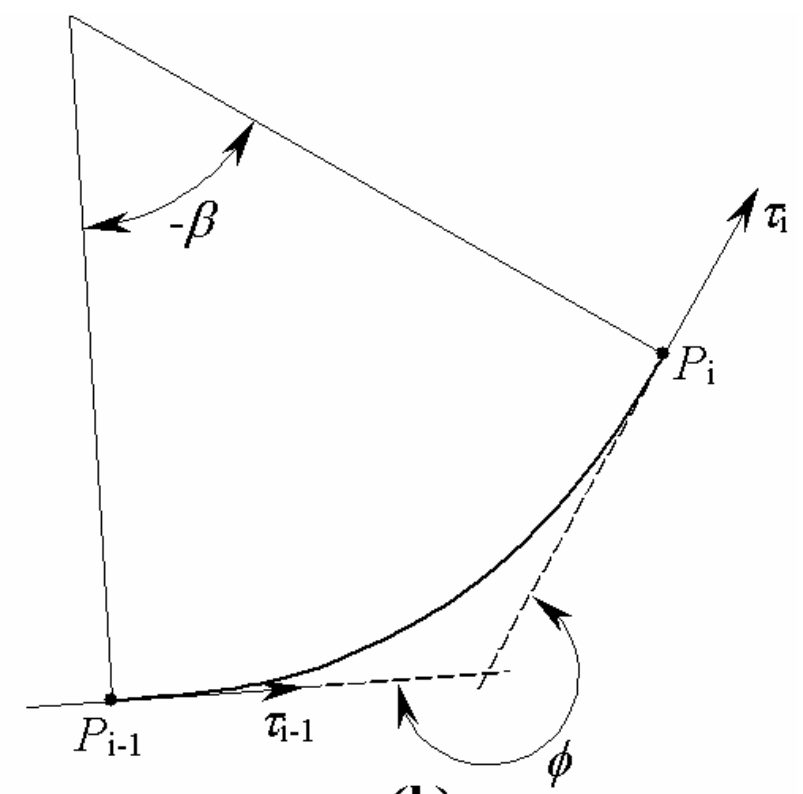

(b)

Fig. 4: Definition of bending angle

This general curve is decomposed of two arc sections $O A$ and $B C$ connected by a straight section $A B$. The global coordinate system is $o x y$. The coordinates of $A, B$, and $C$ are $\mathbf{A}=\left[x_{A}, y_{A}, 1\right]^{T}, \mathbf{B}=$ $\left[x_{B}, y_{B}, 1\right]^{T}$, and $\mathrm{C}=\left[x_{C}, y_{C}, 1\right]^{T}$. The included angle and radius for $O A$ are denoted as $\phi_{O A}$ and radius $r_{O A}$. Those for $B C$ are denoted as $\phi_{B C}$ and radius $r_{B C}$. Because of the process-induced shrinkage and spring-in, points $A, B$, and $C$ change to $A^{\prime}, B^{\prime}$, and $C^{\prime}$.

A tree graph is constructed as shown in Fig. 5. The spring-in angles of each piece are marked on the tree graph. For piece $i$, its spring-in angle $\Delta \phi_{i}$ will cause all the consequent pieces $i+1, i+2, \ldots$, $n$ rotate by $\Delta \phi_{i}$. The dimensional variations of each node are found in a sequential way.

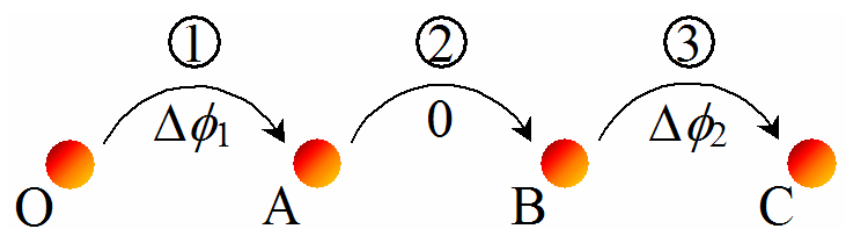

Fig. 5: Tree graph of the example part 
Considering the arc section $O A$, a local coordinate system $o \xi_{1} \eta_{1}$ is constructed by rotating $o x y$ by $\chi_{O A}$. In this case, $\chi_{O A}$ is negative. $\chi_{O A}=\arctan \left(\frac{y_{A}-y_{O}}{x_{A}-x_{O}}\right)$. The length of segment $O A$ is

$$
|O A|=\sqrt{\left(x_{A}-x_{O}\right)^{2}+\left(y_{A}-y_{O}\right)^{2}} .
$$

Thus, the radius of $\operatorname{arc} O A$ is

$$
r_{O A}=\frac{\sqrt{\left(x_{A}-x_{O}\right)^{2}+\left(y_{A}-y_{O}\right)^{2}}}{2 \sin \left(\frac{\beta_{O A}}{2}\right)} .
$$

$r_{O A}$ decreases due to spring-in, i.e.

$$
r_{O A}^{\prime}=\frac{\left(1-\alpha_{T} \Delta T\right) \sqrt{\left(x_{A}-x_{O}\right)^{2}+\left(y_{A}-y_{O}\right)^{2}}}{2 \sin \left(\frac{\beta_{O A}}{2}\right)} .
$$

Thus, in $o \xi_{1} \eta_{1}$, the coordinate of $A^{\prime}$ is

$$
\begin{gathered}
\xi_{A}=\frac{\left(1-\alpha_{T} \Delta T\right) \sqrt{\left(x_{A}-x_{O}\right)^{2}+\left(y_{A}-y_{O}\right)^{2}}}{2 \sin \left(\frac{\beta_{O A}}{2}\right)} \sin \left(\frac{\beta_{O A}^{\prime}}{2}\right) \cos \left(\frac{\Delta \phi_{O A}}{2}\right) \\
\eta_{A}=\frac{\left(1-\alpha_{T} \Delta T\right) \sqrt{\left(x_{A}-x_{O}\right)^{2}+\left(y_{A}-y_{O}\right)^{2}}}{2 \sin \left(\frac{\beta_{O A}}{2}\right)} \sin \left(\frac{\beta_{O A}^{\prime}}{2}\right) \sin \left(\frac{\Delta \phi_{O A}}{2}\right)
\end{gathered} .
$$

The coordinate of $A^{\prime}$ in $o x y$ is

$$
\begin{aligned}
& x_{A}^{\prime}=\xi_{A} \cos \chi_{O A}-\eta_{A} \sin \chi_{O A} . \\
& y_{A}^{\prime}=\xi_{A} \sin \chi_{O A}+\eta_{A} \cos \chi_{O A}
\end{aligned}
$$

Considering the straight section $A B$ in $o x_{1} y_{1}$ with $A$ as the origin, there is no spring-in induced but only shrinkage. If $O A$ has no rotation, the coordinate of $B^{\prime}$ in $o x_{1} y_{1}$ is

$$
\begin{aligned}
& x_{B A}^{\prime}=\left(1+\alpha_{I} \Delta T\right) x_{B A} . \\
& y_{B A}^{\prime}=\left(1+\alpha_{I} \Delta T\right) y_{B A} .
\end{aligned}
$$

Since the spring-in of $O A$ causes all the consequent pieces rotate by $\Delta \phi_{O A}$, the coordinate of $B^{\prime}$ in oxy is

$$
\begin{aligned}
& x_{B}^{\prime}=x_{A}^{\prime}+x_{B A}^{\prime} \cos \Delta \phi_{O A}-y_{B A}^{\prime} \sin \Delta \phi_{O A} \\
& y_{B}^{\prime}=y_{A}^{\prime}+x_{B A}^{\prime} \cos \Delta \phi_{O A}+y_{B A}^{\prime} \cos \Delta \phi_{O A}
\end{aligned} .
$$

Likewise, the coordinate of $C^{\prime}$ in $o x_{2} y_{2}$ is

$$
\begin{aligned}
& x_{C B}^{\prime}=\xi_{C} \cos \chi_{B C}-\eta_{C} \sin \chi_{B C} \\
& y_{C B}^{\prime}=\xi_{C} \sin \chi_{B C}+\eta_{C} \cos \chi_{B C}
\end{aligned} .
$$

The coordinate of $C^{\prime}$ in oxy is

$$
\begin{aligned}
& x_{C}^{\prime}=x_{B}^{\prime}+x_{C B}^{\prime} \cos \left(\Delta \phi_{O A}+\Delta \phi_{A B}\right)-y_{C B}^{\prime} \sin \left(\Delta \phi_{O A}+\Delta \phi_{A B}\right) \\
& y_{C}^{\prime}=y_{B}^{\prime}+x_{C B}^{\prime} \cos \left(\Delta \phi_{O A}+\Delta \phi_{A B}\right)+y_{C B}^{\prime} \cos \left(\Delta \phi_{O A}+\Delta \phi_{A B}\right)
\end{aligned} .
$$

i.e.

$$
\begin{aligned}
& x_{C}^{\prime}=x_{A}^{\prime}+x_{B A}^{\prime} \cos \Delta \phi_{O A}-y_{B A}^{\prime} \sin \Delta \phi_{O A}+x_{C B}^{\prime} \cos \left(\Delta \phi_{O A}+\Delta \phi_{A B}\right)-y_{C B}^{\prime} \sin \left(\Delta \phi_{O A}+\Delta \phi_{A B}\right) \\
& y_{C}^{\prime}=y_{A}^{\prime}+x_{B A}^{\prime} \cos \Delta \phi_{O A}+y_{B A}^{\prime} \cos \Delta \phi_{O A}+x_{C B}^{\prime} \cos \left(\Delta \phi_{O A}+\Delta \phi_{A B}\right)+y_{C B}^{\prime} \cos \left(\Delta \phi_{O A}+\Delta \phi_{A B}\right)
\end{aligned} .
$$

In the vector form, Eqs. 9 through 11 can be rewritten as 


$$
\begin{aligned}
& \mathbf{A}^{\prime}=\mathbf{O A}^{\prime} \\
& \mathbf{B}^{\prime}=\mathbf{O A}^{\prime}+\mathbf{R}_{\mathrm{zOA}} \cdot \mathbf{A}^{\prime} \mathbf{B}^{\prime} \\
& \mathbf{C}^{\prime}=\mathbf{O A}^{\prime}+\mathbf{R}_{\mathrm{zOA}} \cdot \mathbf{A}^{\prime} \mathbf{B}^{\prime}+\mathbf{R}_{\mathrm{zOA}} \cdot \mathbf{R}_{\mathrm{zAB}} \cdot \mathbf{B}^{\prime} \mathbf{C}^{\prime}
\end{aligned}
$$

The dimensional variations of this general curved part were calculated assuming AS4 fiber and epoxy resin. The material properties of AS4 fiber are as shown in Table 1. When the stacking sequence is $\left[0 / 90^{\circ}\right]_{6 s}$, the in-plane and through-thickness CTEs were calculated as

$$
\begin{aligned}
& \alpha_{11}= \begin{cases}4.535 \times 10^{-6} /{ }^{\circ} \mathrm{C} & T=\text { room temperature } \\
2.896 \times 10^{-6} /{ }^{\circ} \mathrm{C} & T=150^{\circ} \mathrm{C}\end{cases} \\
& \alpha_{22}= \begin{cases}54.87 \times 10^{-6} /{ }^{\circ} \mathrm{C} & T=\text { room temperature } \\
54.45 \times 10^{-6} /{ }^{\circ} \mathrm{C} & T=150^{\circ} \mathrm{C}\end{cases}
\end{aligned}
$$

Table 1: Material properties of AS4 fiber

\begin{tabular}{cc}
\hline$E_{f L}(\mathrm{GPa})$ & $E_{f T}(\mathrm{GPa})$ \\
235 & 14 \\
\hline$v_{f}$ & $v_{m}$ \\
0.2 & 0.4 \\
\hline$\alpha_{f L}\left(\mathrm{ppm} /{ }^{\circ} \mathrm{C}\right)$ & $\alpha_{f T}\left(\mathrm{ppm} /{ }^{\circ} \mathrm{C}\right)$ \\
-0.4 & 18 \\
\hline
\end{tabular}

The bending angles are $\beta_{O A}=45^{\circ}, \beta_{A B}=0$, and $\beta_{A B}=-45^{\circ}$. The spring-in angles are calculated as $\Delta \phi_{1}=-0.30^{\circ}, \Delta \phi_{2}=0$, and $\Delta \phi_{3}=0.30^{\circ}$. The dimensional variations of $A$ through $C$ calculated using Eq. 12 are

$$
\Delta \mathbf{A}=\left[\begin{array}{l}
-0.14 \\
-0.16
\end{array}\right] \quad \Delta \mathbf{B}=\left[\begin{array}{l}
-0.42 \\
-0.40
\end{array}\right] \quad \Delta \mathbf{C}=\left[\begin{array}{l}
-0.49 \\
-0.48
\end{array}\right],
$$

where the unit is mm. By using the FEA, the dimensional variations of $A$ through $C$ are found as

$$
\Delta \mathbf{A}=\left[\begin{array}{l}
-0.13 \\
-0.16
\end{array}\right] \quad \Delta \mathbf{B}=\left[\begin{array}{l}
-0.42 \\
-0.39
\end{array}\right] \quad \Delta \mathbf{C}=\left[\begin{array}{l}
-0.49 \\
-0.47
\end{array}\right] .
$$

It shows that a good agreement exists between the STM and FEA.

\section{Conclusions}

A study on the dimensional variations of general curved composite parts is presented in this paper. The spring-in was calculated based on a simple mathematical formula and equivalent material properties. For a general curved composite part, a Structural Tree Method (STM) was developed to divide the curve into a number of pieces and calculate the dimensional variations sequentially. This method can be also applied to an assembly of composite parts. Using the developed STM, the dimension variations of composite components and assemblies can be predicted efficiently. This approach was validated against FEA. The results show that a good agreement exists between the STM and FEA. The method presented in this paper provides the foundation of the tolerance analysis/synthesis for composite components and assemblies.

\section{Acknowledgment}

The author is supported by the Curtin Research Fellowship. 


\section{References}

[1] P. K. Mallick: Fiber-Reinforced Composites: Materials, Manufacturing, and Design, Marcel Dekker, New York, (1993), 2nd edn.

[2] M. J. Shuart, N. J. Johnston, H. B. Dexter, J. M. Marchello and R. W. Grenoble: Tech. Rep. UMI Order Number: NASA-98-AGARD-mjs, NASA Langley Technical Report Server (1998).

[3] H. T. Hahn and N. J. Pagano: Journal of Composite Materials Vol. 9 (1975), p. 91.

[4] D. W. Radford and R. J. Diefendorf: Journal of Reinforced Plastics and Composites Vol. 12 (1993), p. 58.

[5] C. K. Huang and S. Y. Yang: Composites Part A Vol. 28 (1997), p. 891.

[6] L. P. Kollar: Journal of Composite Materials Vol. 28 (1994), p. 392.

[7] L. K. Jain and Y. W. Mai: Journal of Composite Materials Vol. 31 (1997), p. 673.

[8] L. K. Jain, B. G. Lutton, Y. W. Mai and R. Paton: Journal of Composite Materials Vol. 31 (1997), p. 696.

[9] K. J. Yoon and J.-S. Kim: Journal of Composite Materials Vol. 35 (2001), p. 253.

[10] J. Wang, D. Kelly and W. Hillier: Journal of Composite Materials Vol. 34 (2000), p. 1456.

[11] Y. Ding, W. K. Chiu and X. L. Liu: Polymers \& Polymer Composites Vol. 9 (2001), p. 393. 